\title{
Operation modes and control algorithms of anisotropic permanent magnet synchronous motor (IPMSM)
}

\author{
Aleksandr Lutonin ${ }^{1, *}$, Andrey Shklyarskiy ${ }^{1}$, and Yaroslav Shklyarskiy ${ }^{1}$ \\ ${ }^{1}$ Saint-Petersburg Mining University, 2, 21st Line of Vasilevskiy Island, St. Petersburg 199106, Russian Federation
}

\begin{abstract}
This paper represents control strategy of anisotropic permanent magnet synchronous motor (IPMSM) in the field-weakening region. Field weakening controller allows to increase maximum achievable speed with output torque reduction. Proposed control system consists of four general modes: MTPA (maximum torque per ampere), MC (maximum current), FW (field weakening), and MTPV (maximum torque per voltage) which must be chosen accordingly to motor speed, current and torque references. Operation point is found as an intersection of torque hyperbola and voltage ellipse curves in dq motor's current reference frame involving motor parameters' limits. However, due to nonlinear dependence between torque and voltage equations, it is quite complicated to obtain both right control mode selection and reference output calculation. In order to solve this problem, a unified control algorithm adopted for wide speed and torque reference with online constraints calculation is proposed. Matlab/Simulink control model of PMSM motor and control system were designed in order to show developed strategy performance. Simulation results shows increasing of speed limit by more than 2.5 times related to nominal speed with high controller's response. However, speed limit increasing leads to a decrease in motor's output torque. Due to this fact, presented control strategy is not suitable for applications where nominal torque level is essential for all speed operation points.
\end{abstract}

\section{Introduction}

Permanent magnet synchronous motors are widely popular in different industrial applications, such as machinery [1], power generation [2-4], as a source for high current consumers [5,6] with current limiting [7,8] devices, autonomous research units [9], hybrid [10-12], electric [13] vehicle motion, aircraft [14], etc. That popularity take place because of exceptional characteristics in energy efficiency, rotor inertia and power to weight ratio compare to other types of motors [15-16]. In order to achieve speed more the nominal one, field weakening control algorithms are essential. According to [16-17] for wide speed applications anisotropic permanent magnet synchronous motors are more suitable than isotropic ones because of smaller airgap between rotor and stator surfaces, which leads to additional reluctance torque in field weakening operation region [18]. Generally, there are four general operation modes, which must be chosen due to a reference speed, torque values, motor parameters and current, voltage constraints: MTPA (maximum torque per ampere), MC (maximum current), FW (field weakening) and MTPV (maximum torque per voltage), which is detailly presented on figure 1. Also, papers [19-20] describe MTPF (maximum torque per flux) control strategy which is alternative to MPTV. MTPV and MTPF curve shapes are similar to each other, however MTPF strategy's result current references have higher magnitude, so MTPV strategy is more efficient [14].

MTPA strategy, described in [21-22] operates at speeds under the nominal one (curve from 0 to $\mathrm{A}$ on figure 1). MC strategy must be chosen when motor's reference speed and torque are higher than stan standard value (arc which starts from MTPA curve endpoint (point A) and ends in point B which is an intersection of MPTV curve and maximum current circle). MTPV control mode can be used only in "infinite" speed IPMSM motors: motors, where maximum speed limits only by mechanical losses [23]). In finite speed motors point "C" also known as characteristic current [24] locates beyond the maximum current circle. This mode involves to use voltage reference limit as constraint instead of maximum current limit used in MC strategy. It helps to achieve the same torque output with lower current amplitude, compare to MC control. FW - area inside of the shape, limited by three mentioned above strategies (0-A-B-C points).

\footnotetext{
* Corresponding author: lutonin93@mail.ru
} 


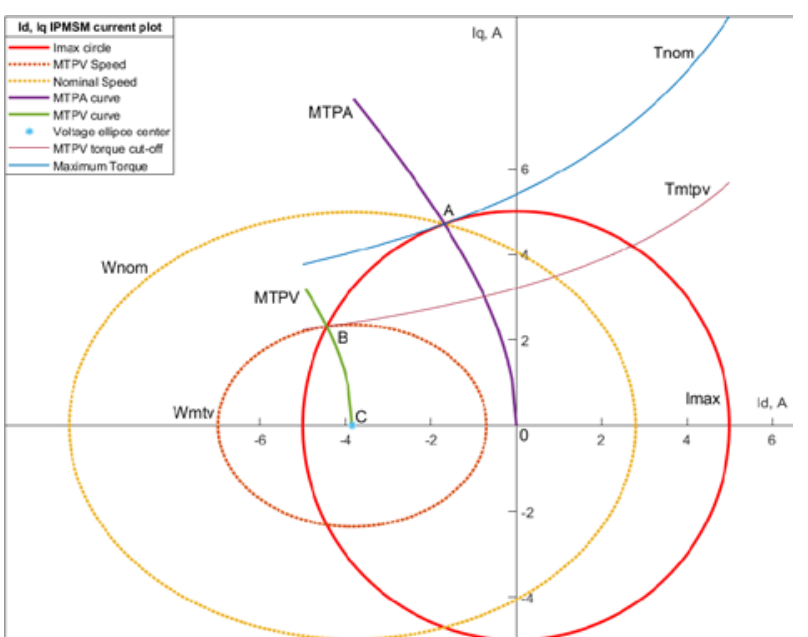

Fig. 1. IPMSM motor d, q axis current plot

\section{Materials and methods}

\subsection{IPMSM mathematical model}

In order to simulate proposed control system design, mathematical model of anisotropic permanent magnet synchronous motor must be obtained. According to [19] IPMSM motor equations can be written as:

$$
\left\{\begin{array}{l}
u_{d}(t)=R \cdot i_{d}(t)+L_{d} \frac{d i_{d}(t)}{d t}-\omega_{m e}(t) \cdot L_{q} \cdot i_{q}(t) \\
u_{q}(t)=R \cdot i_{q}(t)+L_{q} \frac{d i_{q}(t)}{d t}-\omega_{m e}(t) \cdot L_{d} \cdot i_{d}(t)+\omega_{m e}(t) \cdot \psi_{p m} \\
T_{e}(t)=\frac{3}{2} p \cdot\left(\psi_{p m}+\left(L_{d}-L_{q}\right) \cdot i_{d}(t)\right) \cdot i_{q}(t)
\end{array}\right.
$$

where $u_{d}(t) \quad-\mathrm{d}$-axis voltage; $u_{q}(t)-$ q-axis voltage; $R \quad$ - stator resistance; $L_{d}-\mathrm{d}$-axis selfinductance; $L_{q}-$ q-axis self-inductance; $\omega_{m e}-$ electrical speed; $\psi_{p m}$ - permanent magnets flux linkage or field flux linkage; $i_{d}(t)-\mathrm{d}$-axis current; $i_{q}(t)-\mathrm{q}-$ axis current; $T_{e}(t)$ - motor developed torque; $p$-pole pair number;

\subsection{IPMSM motor operation constraints}

Current and voltage constraints can be found according to:

$$
\left\{\begin{array}{c}
i_{d}^{2}+i_{q}^{2} \leq I_{s \max }^{2} \\
u_{d}^{2}+u_{q}^{2} \leq U_{s \max }^{2}
\end{array}\right.
$$

where $I_{s \max }-$ motor's maximum current; $U_{s \max }=\frac{U_{d c}}{\sqrt{3}}--$ maximum output voltage of inverter.

After simplification $u_{d}(t), u_{q}(t)$ equations in (1): neglecting voltage drop on $R$ and taking steady state assumptions e.g. $\frac{d i_{d}(t)}{d t}=0 ; R \cdot i_{d}(t)=0 ; R \cdot i_{q}(t)=0$ and substituting it into (2), the following ellipse voltage constraint according to reference speed value:

$$
\frac{\left(i_{d}+\frac{\psi_{p m}}{L_{d}}\right)^{2}}{\left(\frac{U_{s \max }}{L_{d} \cdot \omega_{m e}}\right)^{2}}+\frac{i_{q}^{2}}{\left(\frac{U_{s \max }}{L_{q} \cdot \omega_{m e}}\right)^{2}} \leq 1
$$

where $\left(-\frac{\psi_{p m}}{L_{d}} ; 0\right)$ - the ellipse center, which value is equal to characteristic current, mentioned above.

For PMSM operation mode computations its more convenient to use the alternative version of (3):

$$
\omega_{m e}=\frac{U_{s \max }}{\sqrt{\left(L_{q} \cdot i_{q}\right)^{2}+\left(\psi_{p m}+L_{d} \cdot i_{d}\right)^{2}}}
$$

\subsection{IPMSM motor operation strategies}

\subsubsection{Maximum torque per ampere strategy (MTPA)}

According to [16-17] MTPA control allow to achieve maximum torque value with minimum torque amplitude. MTPA hyperbola can be found as intersection of voltage ellipse constraint (4) with current circuit area (2):

$$
\begin{aligned}
& i_{d \text { MTPA }}=\frac{\psi_{p m}}{4\left(L_{d}-L_{q}\right)}-\sqrt{\frac{\psi_{p m}^{2}}{16\left(L_{d}-L_{q}\right)^{2}}+\frac{I_{s}^{2}}{2}} \\
& i_{q \text { MTPA }}=\sqrt{I_{s}^{2}-I_{d M T P A}^{2}}
\end{aligned}
$$

where $I_{s}$ - current setpoint amplitude.

This operation mode only achievable until setpoint current amplitude reaches its maximum value $I_{s \max }$. At point $I_{s \max }$ motor reaches its nominal speed $\left(\omega_{\text {nom }}\right)$ and nominal torque values $\left(T_{\text {nom }}\right)$, which can be calculated by substituting $I_{s}$ to $I_{s \max }$, achieving $i_{d \max }$ and $i_{\text {qmax }}$ reference points and substitution this values into (1) and (4) accordingly.

\subsubsection{Maximum current strategy (MC)}

When reference torque and speed is higher than nominal, MC control algorithm is significant. Reference points can be found as intersection of $I_{s \max }$ current circle with voltage ellipse constraint. Therefore, $i_{d M C}$ and $i_{q M C}$ can be found as:

$$
\begin{gathered}
i_{d M C}=\frac{-\psi_{p m} \cdot L_{q}+\sqrt{\left(\psi_{p m} \cdot L_{q}\right)^{2}-\left(L_{d}-L_{q}\right)^{2} \cdot\left(L_{q} \cdot I_{s \max }^{2}+\psi_{p m}^{2}-U_{s \max }^{2} / \omega_{m e}^{2}\right)^{2}}}{4\left(L_{d}-L_{q}\right)} \\
i_{q M C}=\sqrt{I_{s \text { max }}^{2}-I_{d M C}^{2}}
\end{gathered}
$$




\subsubsection{Maximum torque per voltage strategy (MTPV)}

MPTV operation mode requires for deep flux weakening operation in motors, where characteristic current is less than maximum current amplitude. MTPV strategy: power loss minimization subjected to maximum output speed. According to [14] MTPV d and q axis currents can be found as:

$$
\begin{aligned}
& i_{d M T P V}=-\frac{\psi_{M T P V}+\psi_{p m}}{L_{d}} \\
& i_{q \text { MTPV }}=\frac{\sqrt{\frac{U_{s \max }^{2}}{\omega_{m e}^{2}}-\psi_{M T P V}^{2}}}{L_{q}}
\end{aligned}
$$

where $\psi_{M T P V}$ can be found as:

$$
\psi_{\text {MTPV }}=\frac{L_{q} \psi_{p m}-\sqrt{\left(L_{q} \cdot \psi_{p m}\right)^{2}+8 \cdot\left(L_{d}-L_{q}\right)^{2} \cdot \frac{U_{s \max }^{2}}{\omega_{m e}^{2}}}}{4\left(L_{d}-L_{q}\right)}
$$

MTPV control operating region starts on point, which is an intersection of MTPV hyperbola and maximum current circle and ends in characteristic current point $\left(-\frac{\psi_{p m}}{L_{d}} ; 0\right)$, where there is an infinite theoretical speed is achievable.

\subsubsection{Field weakening strategy}

All operation points, which lies inside of described above strategies limitations are FW operating points. $i_{d F W}$ and $i_{q F W}$ reference points can be found as intersection of voltage ellipse circle reference speed and its torque hyperbola. In that case, the reference currents can be found by substituting torque equation into voltage limit constraint equation (4). Result of solving this equation, according to [20] is presented in (12):

$$
\begin{aligned}
& i_{q F W}^{4}+p_{2} \cdot i_{q F W}^{2}+p_{1} \cdot i_{q F W}+p_{0}=0 \\
& p_{2}=\frac{\psi_{p m}^{2} \cdot L_{q}^{2}-\left(L_{d}-L_{q}\right)^{2} \cdot \frac{U_{s \max }^{2}}{\omega_{m e}^{2}}}{L_{q}^{2} \cdot\left(L_{d}-L_{q}\right)^{2}} \\
& p_{1}=\frac{-4 \cdot T_{e} \cdot L_{d} \cdot L_{q} \cdot \psi_{p m}}{3 \cdot L_{q}^{2} \cdot p \cdot\left(L_{d}-L_{q}\right)^{2}} \\
& p_{0}=\frac{\left(2 \cdot T_{e} \cdot L_{d}\right)^{2}}{9 \cdot L_{q}^{2} \cdot p^{2} \cdot\left(L_{d}-L_{q}\right)^{2}}
\end{aligned}
$$

Equation (12) can be solved in MATLAB software package by "solve” function with "MaxDegree, 4". After getting analytical solution $i_{q F W}$, one can find $i_{d F W}$ by substituting $i_{q F W}$ current into voltage constraint equation (4). Therefore, final result is presented in (16):

$$
i_{d F W}=-\frac{\psi_{p m}}{L_{d}}+\frac{1}{L_{d}} \cdot \sqrt{\frac{U_{s \max }^{2}}{\omega_{m e}^{2}}-\left(L_{q} \cdot i_{q F W}\right)^{2}}
$$

\subsection{Proposed control algorithm design}

Proposed control strategy represents on figure 2. Control algorithm structure can be divided into two parts: offline and online parameters computations.

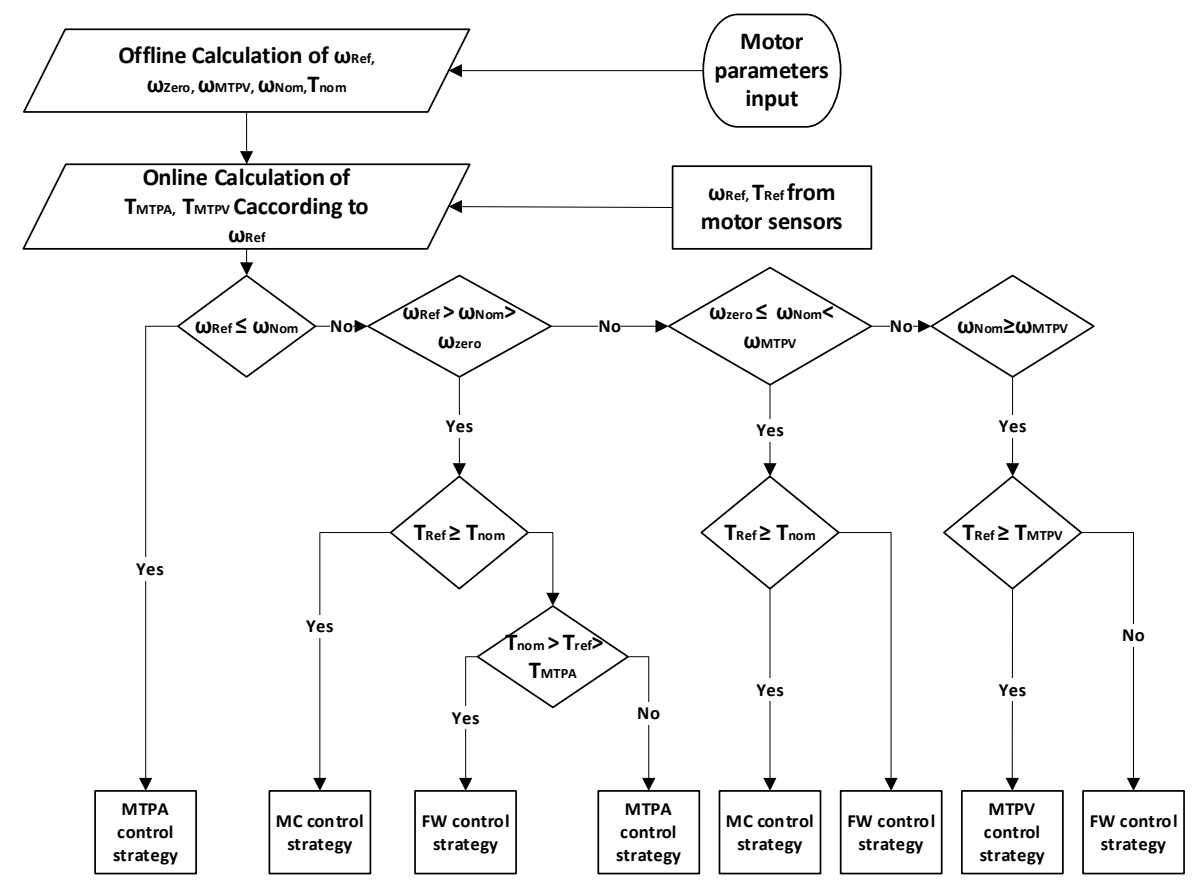

Fig. 2. IPMSM control strategy 
For offline computation main parameters are: $\omega_{\text {zero }}-$ speed reference which crosses $\left(i_{d}=0 ; i_{q}=0\right)$ point; $\omega_{M T P V}$ - speed in intersection of MTPV curve and maximum current circle (point " $\mathrm{B}$ " on figure 1); $T_{\text {nom }}-$ nominal torque value.

In online mode, according to reference speed $\omega_{\text {ref }}$ and reference torque $T_{\text {ref }}$ should be calculated: $T_{\text {MTPV }}-$ torque reference which can be found by equation (1) at point, where an intersection of MTPV locus and $\omega_{\text {ref }}$ voltage ellipse intersection take place; $T_{\text {MTPA }}$ - torque reference at point of intersection of MTPA curve and $\omega_{\text {ref }}$ voltage ellipse.

\section{Results and discussion}

Simulations of proposed control system were provided by Matlab/Simulink simulation software. Simulated motor parameters are following: $U_{s \max }=600 \mathrm{~V} ; I_{\text {s } \max }=$ $5 \mathrm{~A} ; \quad p=3 ; \quad L_{d}=0.008 \mathrm{H} ; \quad L_{q}=0.006 \mathrm{H} ; \psi_{p m}=$ $0.23 \mathrm{~Wb} ; R=20 \mathrm{Ohm}$;

Control block module consists of following blocks: “MTPV, MTPA, MC, FW" block, where operation mode choice take place and calculates $i_{d}, i_{q}$ currents reference values. Current setpoint $I_{s}$ is an output of speed PI regulator. $\omega_{\text {ref }}$ and $T_{\text {ref }}$ - measured speed and torque from motor's sensors. Detailed view of Simulink control block module is presented on figure 3 :

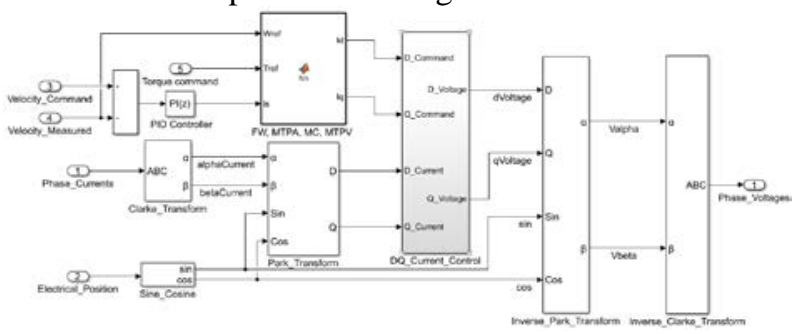

Fig. 3. IPMSM Simulink control block overview.

Figure 4 represents simulation results: according to motor technical specification, nominal motor speed is about $1500 \mathrm{rpm}$. Simulation results shows increasing allowable speed up to 2.5 times. During simulation motor has static torque load with $1.5 \mathrm{Nm}$. From start to end controller worked in all control modes: From 0 to 1500rpm - MTPA control mode. From 1500 to 2600rpm - FW mode; At steady 2600rpm - MC mode; from 2600 to 3900rpm - FW and MTPA mode. Figure 4, except speed reference also shows Voltage and DC current consumption references. Spikes on the plot happens because of tight PI current and voltage regulator settings which was chosen in order to get the best performance in speed characteristic.

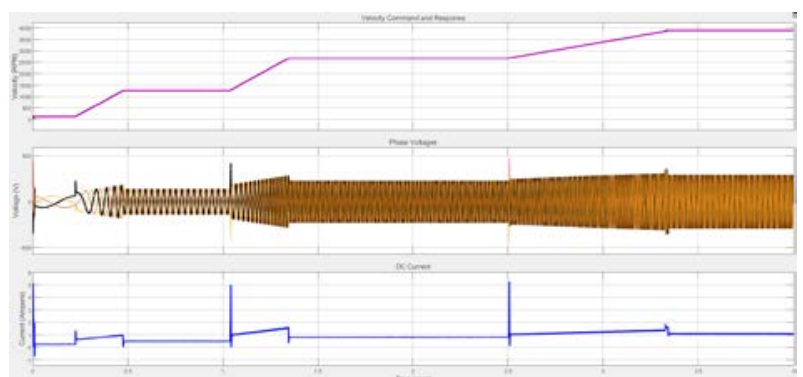

Fig. 4. Simulation results

\section{Conclusions}

In this paper we present control system which allows to reach up to 2.5 times speed increasing due to nominal motor parameters (1500 rpm) with high speed and current controller's response. However, speed increasing leads to output torque falling even up to zero in maximum speed point. Thus, simulated control system can be useful in electric or hybrid vehicle applications, where it is important to have full motor torque capacity at the beginning of motion and high-speed tolerance with less torque limit in dynamic mode. Also, it should be noted that proposed control system must only have been applied on high performance microcontroller systems because all constraints and reference parameters calculates by complicated equations with floating points results.

\section{References}

1.A. Shewy, IJRRAS 4 (2010)

2.P. Boonyang, K. Sittchai, N. Akeratana, Energ. Proceed., 34, 382-389 (2013)

3.J. Zhao, Y. Liu, X. Xu, XIII ICEM, 2437-2443

4. A. Khirtov, A. Khirtov, $10^{\text {th }}$ ISPC, 1, 53-55 (2012)

5. I. Murashov, V. Frolov, A. Kadyrov, Journ. of Phys. Conf. Series, 1058, 1-4 (2018)

6. V. Frolov, I. Matveev, D. Ivanov, S. Zverev, B. Ushin, G. Petrov, Romanian Journ. of Phys, 56, (2011)

7.K. Vostrov, V. Frolov, S. Evgeniy, Plasma Phys. and Tech. Journ. 4, 182-185 (2017)

8. B. Aleksandr, P. Mikhail, V. Leontev, A. Leks, N. Kurakina, A. Kiselev, J. Simakova, V. Frolov, Plasma Phys. and Tech, 4, 120-123 (2017)

9. I. Sobota, V. Malarev,A. Kopteva, Journ. of Mining Inst., 238, 443-449 (2019)

10. S. Mohd, A. Fauzi, K. Ahmad, IJAME, 7, 2229-8649 (2013)

11. G. Mercedes, H. Kay, G. Patrick, WEVJ, 3

12. W. Wang, R. Fu, Y. Fan, $5^{\text {th }}$ IFAC, 51, 407414 (2018)

13. R. Wang, X. Jia, S. Dong, Q. Zhang, $13^{\text {th }}$ IEEE ICIEA, 1733-1738 (2018)

14. M. Villani, M. Tursini, G. Fabri, L. Castellini, IEEE Trans. Ind. Electron, 59, 2073-2081 (2012)

15. F. Demmelmayr, M. Troyer, M. Schroedl, 37th IECON, 2762-2768 (2011) 
16. G. Pellegrino, A. Vagati, B. Boazzo, P. Guglielmi, IEEE Transact. on Ind. Appl., 48, 2322-2332 (2012)

17. H. Murakami, Y. Honda, H. Kiriyama, S. Morimoto, Y. Takeda, 34 ${ }^{\text {th }}$ IEEE IAC, 2, 840-845 (1999)

18. Y. Kim, S. Lee, B. Cho, S. Kwon, Proc. $28^{\text {th }}$ Int. Electr. Vehicle Symp. Esxhibit, 1-7 (2015)

19. H. Eldeeb, C, Hackl, L. Horlbeck, J. Kullick, Int. Journ. of Control, 91, 2273-2302 (2018)

20. Y. Inoue, T. Ichiya, S. Morimoto, $M$. Sanada, IPEC-Hiroshima, 370-375, (2014)

21. M. Preindl, S. Bolognani, IEEE Transact. on Power Electr., 30, 4524-4535 (2015)

22. J. Lemmens, P. Vanassche, J. Driesen, IEEE JESTPE, 2, 249-263 (2014)

23. M. Fadei, L. Sepluchre, M. Pietrzak-David, IFAC, 616-621 (2018)

24. W, Soong, T. Miller, Elec. Power Appl. IEE Proc, 141, 331-340 (1994)

25. O. Tolochko, Energy Efficient Speed Control of Interior Permanent Magnet Synchronous Motor, (2019) 